\title{
The Effect of Daily versus Weekly Levothyroxine Replacement on Thyroid Function Test in Hypothyroid Patients at a Tertiary Care Centre in Haryana
}

\author{
Rajesh Rajput Vaibhav Pathak \\ Department of Endocrinology and Medicine Unit V, Pt. B.D. Sharma Postgraduate Institute of Medical Sciences, \\ Rohtak, India
}

\author{
Keywords \\ Hypothyroidism · Levothyroxine $\cdot$ Weekly administration
}

Conclusion: Once-weekly LT4 administration is a reasonable alternative for patients, especially for those who have issues with compliance.

(C) 2017 European Thyroid Association Published by S. Karger AG, Basel

\begin{abstract}
Objective: We compare the effect of daily versus weekly levothyroxine (LT4) replacement on thyroid function test (TFT) in hypothyroid patients at a tertiary care centre in Haryana. Materials and Methods: This was a randomised crossover study in which 100 hypothyroid patients on a stable LT4 dose were divided into 2 groups of 50 each. In group I, patients were given daily therapy for 6 weeks and then shifted to weekly therapy for the next 6 weeks. In group II, patients were given LT4 once a week for 6 weeks and then switched to daily therapy for the next 6 weeks. TFT, quality of life (QOL), and hyperthyroidism symptom scale (HSS) score of patients were compared in each group at 0,6, and 12 weeks. Results: TFT remained within the normal range, but TSH increased (group I, daily $2.8 \pm 1.4 \mathrm{mIU} / \mathrm{L}$, weekly $3.9 \pm 1.1 \mathrm{mIU} / \mathrm{L}$, $p=0.001$; group II, weekly $4.6 \pm 1.1 \mathrm{mlU} / \mathrm{L}$, daily $2.7 \pm 1.2$ $\mathrm{mlU} / \mathrm{L}, p=<0.001$ ) and T3/T4 decreased with weekly therapy as compared to daily therapy. No significant difference in HSS score was found between daily and weekly administration of LT4. With weekly therapy, QOL showed improvement in bodily pain, vitality, mental health, and social functioning.
\end{abstract}

\section{Introduction}

Hypothyroidism is one of the most common hormone deficiencies with a straightforward diagnosis and treatment as well as an excellent outcome if the patient continues to take treatment regularly. Levothyroxine (LT4) at a dose of $1.6-1.8 \mu \mathrm{g} / \mathrm{kg}$ of body weight per day is the treatment of choice and if taken regularly is sufficient to restore the TSH to the normal range. Around $60-80 \%$ of the ingested dose is absorbed within the first 3-4 h of ingestion [1]. When higher-than-usual doses are needed to maintain TSH in the normal range, clinicians need to find out the reason behind it. One of the most common reasons for poor response to therapy is non-compliance. Non-compliance in hypothyroid patients is due to the need to take the drug daily on an empty stomach. After taking the drug, patients have to wait at least 30 min until they can have a meal, including tea or coffee, to ensure proper absorption of the drug. The need to take the med-

\section{KARGER}

(C) 2017 European Thyroid Association

Published by S. Karger AG, Basel

E-Mail karger@karger.com

www.karger.com/etj
Dr. Rajesh Rajput

Department of Endocrinology and Medicine Unit V PGIMS

Rohtak, Haryana 124001 (India)

E-Mail drrajeshrajput@outlook.com 
ication on a daily basis in the fasting state, to avoid other medications hampering absorption for the next 3-4 h, interferes with the daily routine of the patient and will result in poor compliance which causes poor disease control [2]. To make treatment more patient friendly, various strategies, such as twice-weekly therapy, alternate-day therapy, and once-weekly therapy, have been tried in the literature [3-5]. Since the elimination half-life of LT4 is about 7 days, and its biological effect may last longer, giving it once weekly seems a logical alternative [6]. Also, LT4 is a prohormone which is converted in the body tissues into metabolically active TT3 by the local deiodinase enzyme. In the peripheral tissue, there is autoregulation in the conversion of T4 to T3, which increases at a low T4 level and decreases at a higher T4 level [7]. These properties of LT4 suggest the possibility of using it at a longer dosing interval than the traditional daily dosing. Also, weekly dosing may not only improve compliance in patients but could also be advantageous to nurses or other caregivers taking care of patients who are unable to dose themselves. Grebe et al. [4] found that weekly LT4 replacement was well tolerated with no evidence of toxicity. In their study, the mean serum TSH before the administration of weekly T4 was higher (weekly, $6.61 \mathrm{mIU} / \mathrm{mL}$; daily, $3.92 \mathrm{mlU} / \mathrm{mL} ; p=0.0001$ ), and mean FT4 (weekly, $0.98 \mathrm{ng} / \mathrm{dL}$; daily, $1.35 \mathrm{ng} / \mathrm{dL} ; p=0.01$ ) and FT3 (weekly, 208 pg/dL; daily, 242 pg/dL; $p=0.01$ ) were lower. Apart from few published studies, no study has compared daily with weekly administration of LT4 in Indian hypothyroid patients. In view of these facts, the present study was planned to compare the effect of daily and weekly LT4 replacement on thyroid function test (TFT) and quality of life (QOL) at a tertiary care hospital in Haryana, India.

\section{Materials and Methods}

This was a randomised crossover study including 100 hypothyroid patients attending the Endocrinology Outpatient Department of Pt. B.D. Sharma PGIMS, Rohtak. All of these patients were biochemically euthyroid on stable doses of LT4 for at least 3 months before recruitment into the study. Using a random number table, patients were divided into 2 groups of 50 each. Written informed consent was obtained from all patients, and the institutional ethics committee approved the study. Pregnant and postpartum females as well as patients with co-morbid illnesses, such as diabetes mellitus, hypertension, or coronary artery disease, were not included in the study. At baseline (day 0), patients in group I were advised to continue with their daily LT4, while patients in group II were given 7 times the daily dose once every week. After 6 weeks, patients were advised to switch their therapeutic regimen (i.e., group I was administered 7 times the daily dose once every week and group II was advised to take daily LT4 for another 6 weeks). The

Daily vs. Weekly LT4 Replacement in

Hypothyroid Patients
Table 1. Baseline characteristics of study groups I and II

\begin{tabular}{lccl}
\hline Variable & Group I & Group II & $p$ value \\
\hline Age, years & $36.1 \pm 10.7$ & $35.4 \pm 8.4$ & 0.749 \\
Weight, kg & $64.4 \pm 10.1$ & $64.5 \pm 9.0$ & 0.975 \\
Height, cm & $156.2 \pm 5.8$ & $156.9 \pm 5.8$ & 0.513 \\
BMI & $26.4 \pm 3.8$ & $26.1 \pm 3.0$ & 0.700 \\
Pulse, bpm & $77.5 \pm 8.4$ & $77.8 \pm 7.2$ & 0.849 \\
SBP, mm Hg & $120.8 \pm 7.1$ & $119.8 \pm 8.0$ & 0.296 \\
DBP, mm Hg & $77.4 \pm 6.0$ & $76.1 \pm 5.6$ & 0.272 \\
T3, nmol/L & $2.2 \pm 0.3$ & $2.1 \pm 0.4$ & 0.162 \\
T4, nmol/L & $115.3 \pm 19.3$ & $120.1 \pm 16.7$ & 0.243 \\
TSH, mIU/L & $2.5 \pm 1.4$ & $2.6 \pm 1.4$ & 0.638 \\
\hline
\end{tabular}

Data are expressed as mean $\pm \mathrm{SD}$. bpm, beats per minute; SBP, systolic blood pressure; DBP, diastolic blood pressure.

dose of LT4 was not changed during the study period. Patients were studied based on change in TFT and QOL during the 12-week study period. Assessment of TFT was done at baseline (i.e., before randomisation) and after 6 and 12 weeks. T3 (normal range 1.07$3.08 \mathrm{nmol} / \mathrm{L}$ ) and T4 (normal range 70.80-173.70 nmol/L) were assessed by using radioimmunoassay methods (RIAK 4/4A for T3 and RIAK 5/5A for T4). Serum TSH levels (normal range 0.3-5.0 $\mathrm{mIU} / \mathrm{L}$ ) were measured by using an immunoradiometric assay method (IRMAK9). Using SF-36 at baseline, 6 weeks, and 12 weeks, change in QOL was assessed [8]. The scale score ranged from 0 to 100 for 8 health concepts, with higher values indicating a better health status. Clinical changes were evaluated using the hyperthyroidism symptom scale (HSS) [9].

TFT, QOL, and HSS score of patients in both groups were compared at 6 and 12 weeks to find out differences between daily and weekly LT4 administration. A total of 5 patients ( 3 in group I and 2 in group II) were lost to follow-up. Statistical analysis was performed on 95 patients using SPSS software, Version 20.0. A $p$ value of $<0.05$ was considered significant. All investigators measuring the subjective scores were blinded. This study was conducted at a teaching hospital institution, and registration was not mandatory to conduct the study.

\section{Results}

Baseline characteristics of both groups were comparable and are summarised in Table 1. The mean age of the subjects was $36.1 \pm 10.7$ years in group I and $35.4 \pm 8.4$ years in group II $(p=0.749)$ with a male to female ratio of 1:9 in group 1 and 1:7.3 in group II. There was no statistically significant difference in body weight and BMI between the groups. At baseline (i.e., at the time of randomisation, week 0), T3, T4, and TSH were comparable in both study groups with no statistical difference (Table 1). In group I, patients were advised to continue taking 
Table 2. Intragroup comparison of thyroid function test of group I and group II at baseline and after 6 and 12 weeks

\begin{tabular}{|c|c|c|c|c|c|c|}
\hline & baseline (A) & 6 weeks (B) & 12 weeks (C) & baseline (A) & 6 weeks (B) & 12 weeks (C) \\
\hline $\mathrm{T} 4, \mathrm{nmol} / \mathrm{L}$ & $\begin{array}{l}115.3 \pm 19.3 \\
\mathrm{~A}-\mathrm{B}(p=0.120)\end{array}$ & $\begin{array}{l}107.6 \pm 30.5 \\
B-C(p=0.008)\end{array}$ & $\begin{array}{l}94.7 \pm 20.6 \\
\text { C-A }(p \leq 0.001)\end{array}$ & $\begin{array}{l}120.1 \pm 16.7 \\
\mathrm{~A}-\mathrm{B}(p \leq 0.001)\end{array}$ & $\begin{array}{l}90.9 \pm 15.7 \\
\mathrm{~B}-\mathrm{C}(p<0.001)\end{array}$ & $\begin{array}{l}120.1 \pm 15.6 \\
\mathrm{C}-\mathrm{A}(p=0.981)\end{array}$ \\
\hline
\end{tabular}

Data are expressed as mean $\pm \mathrm{SD}$.

LT4 daily until week 6, when their TFT was repeated and they were switched to weekly LT4 admiration. With weekly LT4 administration, there was a decrease in T3 and T4 levels and an increase in TSH as compared to baseline and daily dosing. T3 decreased to $1.8 \pm 0.2$ $\mathrm{nmol} / \mathrm{L}$ at 12 weeks after 6 weeks of weekly therapy as compared to $2.1 \pm 0.4 \mathrm{nmol} / \mathrm{L}$ at 6 weeks and $2.2 \pm 0.3$ $\mathrm{nmol} / \mathrm{L}$ at baseline $(p<0.001)$. The T4 level also decreased significantly when patients were given weekly LT4 as compared to daily dosing both at baseline and at 6 weeks of daily dosing $(p<0.05)$. TSH was $2.5 \pm 1.4 \mathrm{mIU} / \mathrm{L}$ at the time of randomisation (i.e., week 0 ), $2.8 \pm 1.4 \mathrm{mIU} / \mathrm{L}$ after 6 weeks of daily therapy, and when subjects were switched to weekly LT4 therapy it increased to $3.9 \pm 1.1 \mathrm{mIU} / \mathrm{L}$ $(p \leq 0.001)$ (Table 2).

In group II, after measuring TFT, QOL, and HSS score in week 0 , patients were advised to take LT4 once a week for 6 weeks. Then, their TFT was repeated and they were switched back to daily LT4 administration for the next 6 weeks. TFT, SF-36, and HSS score were again measured at 12 weeks and were compared to the values at 6 weeks when patients were taking LT4 on a weekly basis. T3 decreased to $1.7 \pm 0.2 \mathrm{nmol} / \mathrm{L}$ after 6 weeks of weekly therapy from $2.1 \pm 0.4 \mathrm{nmol} / \mathrm{L}$ at baseline and increased again to $2.1 \pm 0.3 \mathrm{nmol} / \mathrm{L}$ when they were switched back to daily therapy; this change was statistically significant $(p \leq$ $0.001)$. The T4 level also decreased to $90.9 \pm 15.7 \mathrm{nmol} / \mathrm{L}$ after 6 weeks of weekly LT4 therapy from $120.1 \pm 16.7$ $\mathrm{nmol} / \mathrm{L}$ at baseline and increased again to $120.1 \pm 15.6$ $\mathrm{nmol} / \mathrm{L}$ when they were switched back to daily therapy; this change was statistically significant $(p \leq 0.001)$. The mean TSH level after weekly LT4 replacement increased to $4.6 \pm 1.1 \mathrm{mIU} / \mathrm{L}$ from a baseline value of $2.6 \pm 1.4$ $\mathrm{mIU} / \mathrm{L}$, and when again switched to daily therapy it was
$2.7 \pm 1.2 \mathrm{mIU} / \mathrm{L}$. When the mean TSH level after weekly therapy and that after daily therapy were compared it was significant statistically $(p \leq 0.001)$ (Table 2 ).

On intergroup comparison after 6 weeks, T3, T4, and TSH levels were significantly different between group I (daily LT4) and group II (weekly LT4). A similar change in trend in TFT was observed even at 12 weeks between the 2 groups when group I was switched to weekly therapy while group II was switched to daily administration of LT4 (Table 3).

During the study, we assessed patients clinically for signs and symptoms of hyperthyroidism using the HSS score. In group I, it was $5.6 \pm 2.2$ after daily LT4 replacement, while after 6 weeks of weekly replacement it was 5.4 \pm 2.6 . When both were compared, there was no statistical difference $(p=0.192)$. Similarly, in group II when HSS scoring was compared for weekly and daily therapy, there was no statistical significance. The HSS score was $6.4 \pm$ 2.3 after weekly therapy and $6.4 \pm 2.8$ after daily therapy $(p=0.771)$. No significant difference in HSS score was found between daily and weekly administration of LT4 in both groups. During our study, we observed that in group I there was a slight improvement in the scores of various domains of SF-36 when patients were administered weekly therapy. When the mean scores of various domains of SF-36 after daily therapy were compared to those after weekly therapy, there was no statistical difference except for "bodily pain," which showed a significant difference $(p=0.007)$. In group II, when the mean score after daily therapy was compared to that after weekly therapy, there was a statistical difference in "vitality" $(p=0.001)$, "social functioning" ( $p=0.001)$, and "mental health" $(p=0.001)$. Other domains in group II did not show a statistical difference $(p>0.005)$. 
Table 3. Intergroup comparison of thyroid function test between group I and group II at 6 and 12 weeks

\begin{tabular}{|c|c|c|c|c|c|c|}
\hline & $\begin{array}{l}\text { group I } \\
\text { (taking LT4 daily) }\end{array}$ & $\begin{array}{l}\text { group II } \\
\text { (taking LT4 weekly) }\end{array}$ & $\begin{array}{l}p \\
\text { value }\end{array}$ & $\begin{array}{l}\text { group I } \\
\text { (taking LT4 weekly) }\end{array}$ & $\begin{array}{l}\text { group II } \\
\text { (taking LT4 daily) }\end{array}$ & $\begin{array}{l}p \\
\text { value }\end{array}$ \\
\hline $\mathrm{T} 3, \mathrm{nmol} / \mathrm{L}$ & $2.1 \pm 0.4$ & $1.7 \pm 0.2$ & $\leq 0.001$ & $1.8 \pm 0.2$ & $2.1 \pm 0.3$ & 0.001 \\
\hline $\mathrm{T} 4, \mathrm{nmol} / \mathrm{L}$ & $107.6 \pm 30.5$ & $90.9 \pm 15.7$ & $\leq 0.001$ & $94.7 \pm 20.6$ & $120.1 \pm 15.6$ & $\leq 0.001$ \\
\hline TSH, mIU/L & $2.8 \pm 1.4$ & $4.6 \pm 1.1$ & 0.001 & $3.9 \pm 1.1$ & $2.7 \pm 1.2$ & $\leq 0.001$ \\
\hline
\end{tabular}

\section{Discussion}

In the present study, weekly therapy with LT4 was found to be as effective as conventional daily therapy in maintenance of euthyroidism. However, a slight decrease in T3 and T4 as well as an increase in the TSH level were observed just before the next due weekly dose. Patients did not develop hyperthyroidism during weekly administration of LT4 as assessed by HSS. SF-36 scores showed significant improvement in bodily pain, vitality, social functioning, and mental health with weekly therapy as compared to daily administration of LT4.

There are very few studies worldwide comparing daily versus weekly administration of LT4 in hypothyroid patients, and none from India. In a study on 14 patients, Bornschein et al. [10] showed similar results and did not observe any hyperthyroidism during weekly therapy. A study done on Asian hypothyroid patients by Doraiswamy and Zainudin [11] demonstrated that after 3 months, there were no significant differences in thyroid profile between the weekly and daily groups. In a case series of 2 patients, Rangan et al. [12] showed that once-weekly T4 is an alternative treatment strategy to treat noncompliant patients and commented that once-weekly T4 is a safe treatment regimen. However, Grebe et al. [4] showed that weekly therapy was well tolerated, but TFT showed mild hypothyroidism with an increase in TSH and a decrease in T3 and T4 before the next weekly dose. Our subjects also showed a slight decrease in T3 and T4, with an increase in TSH with the weekly dose, but the mean TSH in both of our study groups remained within the reference range at all times. Pharmacogenomic studies have suggested that the polymorphism in genes for deiodinase enzyme may be responsible for the varied response to LT4 treatment among hypothyroid patients. Type 2 deiodinase is a major activating enzyme that is responsible for the

Daily vs. Weekly LT4 Replacement in

Hypothyroid Patients conversion of T4 to metabolically active T3. The common Thr92Ala D2 polymorphism has been associated with traits of impaired thyroid hormone action at various endorgan targets, the hypothalamic-pituitary level, and in circulating levels of thyroid hormones [13-15]. Although we have not assessed the peripheral deiodinase activity, it is likely that these genetic differences of deiodinase enzyme activity might be responsible for sufficient peripheral conversion of LT4 to T3 and which would have resulted in maintenance of euthyroidism in our study group despite the rise in TSH level in the weekly group. Similar observations were reported by Grebe et al. [4]. In their study, they found that after the weekly dose of LT4, there was a 3 -fold increase in $\mathrm{fT} 4$, a $50 \%$ increase in the metabolically inactive form rT3, and only a $25 \%$ increase in fT3, but at the end of the dosing interval, fT 4 was $30 \%$ lower, fT3 was $15 \%$ lower, and rT3 was $18 \%$ lower. This suggests that at the end of the weekly treatment, conversion of T4 to T3 increased, and conversion to rT3 decreased. This mechanism may be responsible for the maintenance of euthyroidism while on weekly LT4. Another likely mechanism that may be responsible for maintaining euthyroidism with weekly dosage is that a higherthan-usual level of LT4, when given intermittently, will suppress the thyroid antibody levels. This was demonstrated by Altunta et al. [3] who compared daily with twice-weekly therapy. During their study, they found that higher-than-usual daily doses of LT4 given intermittently may effectively suppress thyroid antibody levels. However, we could not confirm these hypotheses in our study groups as we did not sample subjects for rT3 and TSH levels during the interval between weekly LT4 doses and we did not measure TPO antibody levels at different points of time during the study.

The weekly administration of T4 may cause hyperthyroidism-like symptoms and signs during the initial few 
days of therapy and in the long term can have a detrimental effect on the heart, muscles, and bone. In the present study, we assessed the patients clinically for signs and symptoms of hyperthyroidism using the HSS score and found that it was not suggestive of hyperthyroidism during the entire study period in both groups. However, we did not assess 24 -h electrocardiogram monitoring, echocardiography, as well as bone and muscle parameter measurements which need to be assessed in future studies. The available literature suggests that weekly LT4 is safe from a cardiac point of view; however, due to the small number of patients and short follow-up in those studies, firm safety data for weekly therapy have not been established $[4,11]$.

Clinicians do not routinely assess patients' perceptions of their health status, but it is increasingly being argued that the patient's perspective is as valid as that of the clinician in the evaluation of a chronic medical illness like hypothyroidism. A substantial number of patients on LT4 replacement report not feeling well despite having TFT within the healthy reference range [16]. The same was observed in the present study, where the mean score of various domains of SF-36 was in the range of 50-60 only, despite the fact that all patients were biochemically euthyroid. Weekly therapy showed improvement in various domains of SF-36, such as bodily pain, vitality, mental health, and social functioning. The significant improve- ment in these parameters could be due to a feeling of wellbeing as the burden and cumbersome process of taking medicine on a daily basis is not required when on weekly therapy. However, patients were not blinded from SF-36 measurement. Hence, knowledge and expectations associated with a new treatment could have influenced the scores of SF-36.

The main strengths of the present study include its large sample size and crossover design. All previous studies done so far included few patients. The crossover design allowed us to analyze results not only between groups, but also within the groups, comparing every patient with him/herself, thus minimizing possible confounding factors. However, further studies involving a larger number of patients and a longer duration of follow-up are needed to firmly establish the role of weekly LT4 in the management of hypothyroidism. In conclusion, the present study shows that in today's era of busy and hectic schedules where it is difficult for patients to take LT4 following a rigid schedule, once weekly LT4 administration provides an alternative approach for treatment of hypothyroidism, especially in patients where compliance is a major issue.

\section{Disclosure Statement}

The authors declare no conflicts of interest.

\section{References}

1 Roberts CG, Ladenson PW: Hypothyroidism. Lancet 2004;363:793-803.

2 Mandel SJ, Brent GA, Larsen PR: Levothyroxine therapy in patients with thyroid disease. Ann Intern Med 1993;119:492-502.

3 Altunta F, Uysal AR, Erol C: Twice weekly LT4 for the treatment of primary hypothyroidism. Turk J Endocrinol Metab 2004;1:2534.

4 Grebe SKG, Cooke RR, Ford HC, Fagerström JN, Cordwell DP, Lever NA, et al: Treatment of hypothyroidism with once weekly thyroxine. J Clin Endocrinol Metab 1997;82:870875.

5 Dayal D, Saini L, Attri SV, Singh B, Bhalla AK: Daily versus alternate day thyroxine therapy to maintain euthyroidism in children with congenital hypothyroidism. Int J Endocrinol Metab 2013;1:e9499.

6 Stock JM, Surks MI, Oppenheimer JH: Replacement dosage of L-thyroxine in hypothyroidism. A re-evaluation. N Engl J Med 1974; 290:529-533.
7 Lum SM, Nicoloff JT, Spencer CA, Kaptein EM: Peripheral tissue mechanism for maintenance of serum triiodothyronine values in a thyroxine-deficient state in man. J Clin Invest 1984;73:570-575.

8 Ware JE, Sherbourne CD: The MOS 36-item short-form health survey (SF-36). Med Care 1992;30:473-483.

-9 Klein I, Trzepacz PT, Roberts M, Levey GS: Symptom rating scale for assessing hyperthyroidism. Arch Intern Med 1988;148:387-390.

-10 Bornschein A, Filho GP, Graf H, Gisah A, Carvalh D: Treating primary hypothyroidism with weekly doses of levothyroxine: a randomized, single-blind, crossover study. Arq Bras Endocrinol Metab 2012;56:4.

11 Doraiswamy A, Zainudin S: Efficacy and cardiac safety of short term weekly levothyroxine administration in hypothyroid patient on replacement therapy. ICE/ENDO, Chicago, 2014; Poster Board LBSA-0571.

12 Rangan S, Tahrani AA, Macleod AF, Moulik PK: Once weekly thyroxine treatment as a strategy to treat non-compliance. Postgrad Med J 2007;83:e3.
13 Panicker V, Saravanan P, Vaidya B, Evans J, Hattersley AT, Frayling TM, et al: Common variation in the $\mathrm{DIO} 2$ gene predicts baseline psychological well-being and response to combination thyroxine plus triiodothyronine therapy in hypothyroid patients. J Clin Endocrinol Metab 2009;94:1623-1629.

14 Torlontano M, Durante C, Torrente I, Crocetti U, Augello G, Ronga G, et al: Type 2 deiodinase polymorphism (threonine 92 alanine) predicts L-thyroxine dose to achieve target thyrotropin levels in thyroidectomized patients. J Clin Endocrinol Metab 2008;93:910-913.

15 Peeters RP, van Toor H, Klootwijk W, de Rijke YB, Kuiper GG, Uitterlinden AG, et al: Polymorphisms in thyroid hormone pathway genes are associated with plasma TSH and iodothyronine levels in healthy subjects. J Clin Endocrinol Metab 2003;88:2880-2888.

16 Saravanan P, Chau WF, Roberts N, Vedhara K, Greenwood R, Dayan CM: Psychological well-being in patients on "adequate" doses of L-thyroxine: results of a large, controlled community-based questionnaire study. Clin Endocrinol (Oxf) 2002;57:577-585. 